\title{
Communication-Aware Surveillance in Mobile Sensor Networks
}

\author{
Alireza Ghaffarkhah and Yasamin Mostofi
}

\begin{abstract}
In this paper, we consider a surveillance problem, where a number of mobile nodes are tasked with surveying an area for the possible presence of stationary targets and reporting their findings back to a fixed base station, in the presence of realistic fading communication channels. We develop a mathematical framework for robust communication-aware surveillance, in order to survey the environment efficiently while maximizing the probability of connectivity of the nodes to the base station at all the time. More specifically, we show how to design local motion planning strategies that properly integrate both sensing and communication goals. By using Chernoff bound on the probability of detection error, we prove that the motion planning objective can be separated into a sensing function that maximizes the Kullback-Leibler (KL) divergence between the maximum uncertainty state and the current one, and a communication function, that maximizes the probability of being connected. The resulting motion trajectories provide the right balance between sensing and communication objectives and demonstrate interesting tradeoffs. Our simulation results then show the performance of our proposed communication-aware surveillance framework.
\end{abstract}

\section{INTRODUCTION}

Over the past few years, considerable progress has been made in the area of mobile wireless sensor networks (MWSNs). Applications include environment surveillance, structure monitoring, active coverage, oceanographic sampling and military reconnaissance [1]-[4]. Communication plays a key role in the overall performance of a MWSN as the nodes rely on receiving information from others in order to achieve their task. Due to the already existing complexity of control of robotic networks, however, it is common to assume ideal or over-simplified communication links. Such simplified models may not suffice for ensuring robust group operation and maintaining the connectivity of a MWSN. This necessitates considering realistic link models and devising an integrative approach to communication and motion planning, in order to take into account channel variations when planning the motion.

More recently, a number of papers have started to highlight the importance of considering realistic communication links in cooperative control scenarios [5]-[7]. In our previous work in [5], [6], we considered tracking and maintaining a fixed distance to a moving vehicle by communicating over realistic communication links, showing the importance of considering both communication and navigation objectives.

This work is supported in part by ARO CTA MAST project \# W911NF08-2-0004 and NSF award \# 0812338.

A. Ghaffarkhah and Y. Mostofi are with the Cooperative Network Lab, Department of Electrical and Computer Engineering, University of New Mexico, Albuquerque, NM 87113, USA \{alinem, ymostofi\}@ece.unm. edu
In this paper, we extend our previous work and study a multirobot surveillance problem using a MWSN. We consider a number of mobile sensors that are tasked with surveying an area, for the possible presence of targets, and reporting their findings back to a fixed base station, by communicating over realistic communication channels that experience path loss, shadow fading and multipath fading.

In the communication literature, surveillance networks have been mainly considered with an emphasis on cooperative signal processing considering fading channels [8][10], but without considering motion optimization and navigation. On the other hand, the robotics/control community has looked at robotic surveillance problems, mainly from the perspective of navigation/control of motion, emphasizing sensing objectives [3], [11]-[13]. In this paper, we develop a framework for communication-aware surveillance in realistic communication environments, where each node considers the impact of its motion decisions on both its sensing and communication qualities and optimizes its trajectory accordingly. This is a challenging task and requires 1) assessing wireless link qualities at places that are not yet visited by the robots and 2) proper integration of communication and sensing goals such that each robot chooses a trajectory that provides the best balance between communication and sensing, considering imperfect wireless links. In our previous work in [5], [14], we proposed a probabilistic framework for online channel assessment, based on a small number of a priori wireless measurements. In this paper, we integrate that framework with motion planning and propose a communication-aware surveillance strategy that enables each node to explore the area the best possible (in order to minimize the overall probability of detection error at the base station), while maximizing the probability of its connectivity to the base station. We prove that the overall motion optimization problem, in this case, can be separated into a sensing function that maximizes the Kullback-Leibler (KL) divergence [15] between the maximum uncertainty state and the current one, and a communication function that maximizes the probability of being connected.

The rest of the paper is organized as follows. In Section II, we describe our system model and briefly summarize the probabilistic multi-scale modeling of a channel. In Section III, we mathematically characterize target detection quality of both mobile nodes and the base station. We then propose communication-aware navigation strategies and explore the underlying tradeoffs in Section IV. We present our simulation results in Section V, followed by conclusions in Section VI. 


\section{Problem Formulation}

Consider a scenario where a remote fixed station (to which we refer as a base station) needs to survey a workspace $\mathcal{W} \subset \mathbb{R}^{2}$ for the possible presence of a number of fixed targets (the total number of targets is assumed unknown). The targets, for instance, could be mines that are hidden over a field. The base station uses a team of $n$ mobile robots, which are tasked to survey the workspace and report the locations of the targets. The robots are equipped with sensors, which are able to sweep circular regions around them for the possible presence of targets. The outputs of the local sensors, however, are not perfect and the quality of sensing at a specific position is degraded as its distance to a sensor becomes larger. Each node uses a (local) detection algorithm to fuse its overall gathered data, at any time, and build a discrete binary map for the presence of the targets in the environment, where one (zero) at any position indicates the presence (absence) of the target at that position. The nodes then send their updated binary maps to the base station, which fuses its received data and builds a more precise binary map of the targets. ${ }^{1}$

Without loss of generality, we assume a discretized gridlike workspace that consists of a number of cells. Any position $q \in \mathcal{W}$ then refers to the position of a cell in the discretized workspace. We furthermore assume that the cells are small enough such that there exists at most one target at any cell. The communication links between the nodes and the base station are assumed realistic wireless channels. The dynamics of the links affect the reception of the base station (and as a direct result the performance of the fusion algorithm) in two ways:

- The base station may drop a received packet in case of poor reception.

- The packets that are kept may not be fully error-free due to fading and receiver thermal noise.

Then, the overall goal is for the base station to have the most reliable map of the targets at any time, which requires connectivity maintenance. Fig. 1 shows a schematic of the considered surveillance scenario.

The trajectories of the robots affect both their sensing qualities and received Signal-to-Noise Ratios (SNRs) at the base station, impacting the overall detection quality of the base station. In this paper, we propose a novel communicationaware navigation framework that aims to minimize the overall uncertainty at the base station, while maximizing the probability of being connected. We furthermore show that the optimum trajectories are the ones that provide the right balance between sensing and communication of the robots, given system constraints.

\section{A. Sensing and Dynamical Models of Mobile Sensors}

Let $H_{0}$ and $H_{1}$ denote two hypothesis corresponding to the absence and presence of a target at position $q \in \mathcal{W}$. The

\footnotetext{
${ }^{1}$ Note that in many applications, the nodes can not afford to send their raw measurements to the base station due to constraints on the available resources such as power and bandwidth [9], [10]. Thus, we assume that the robots communicate their updated binary maps.
}

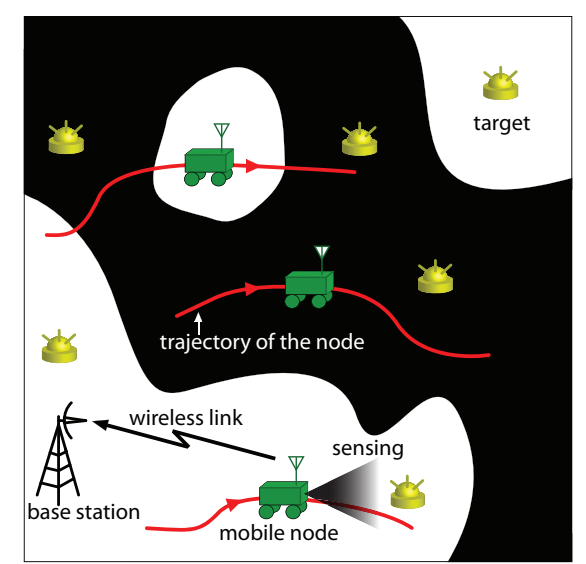

Fig. 1. A schematic of the surveillance scenario considered in this paper. The nodes are not connected to the base station in the black areas, i.e. the received SNR in the black areas is below an acceptable threshold.

observation of the $i^{\text {th }}$ node is then given as follows:

$$
\begin{aligned}
& H_{0}: y_{i, k}(q)=v_{i, k}(q) \\
& H_{1}: y_{i, k}(q)=A+v_{i, k}(q),
\end{aligned}
$$

where $y_{i, k}(q) \in \mathbb{R}$ is the observation of the $i^{\text {th }}$ node at position $q \in \mathcal{W}$ and at time instant $k \geq 1, A>0$ is a positive constant and $v_{i, k}(q) \in \mathbb{R}$ is a zero-mean white Gaussian noise representing the effect of sensing error. The variance of $v_{i, k}(q)$ is given by $\sigma_{i, k}^{2}(q)=\Psi_{i}\left(\left\|q_{i, k}-q\right\|\right)$, where $q_{i, k}$ is the position of the $i^{\text {th }}$ node at time instant $k \geq 1,\|$.$\| represents the Euclidean norm and \Psi_{i}($.$) is a$ positive function [11], [12]. To keep our analysis general, we do not make any assumption on the form of $\Psi_{i}($.$) other$ than the fact that $\Psi_{i}(d)$ is positive and an increasing function of its argument. Each node also has a limited sensing range, such that any target located outside this range simply can not be sensed. Let $\mathcal{S}_{i, k}=\left\{q \in \mathcal{W} \mid\left\|q_{i, k}-q\right\|<d_{i, \text { sen }}\right\}$ denote the sensing region of the $i^{\text {th }}$ node at time instant $k \geq 1$, with $d_{i \text {,sen }}$ representing its sensing radius. Then, we have $\Psi_{i}\left(\left\|q_{i, k}-q\right\|\right) \rightarrow \infty$ for $q \in \mathcal{W} \backslash \mathcal{S}_{i, k}$. As for the dynamics of the robots, we consider the following general discrete-time form $q_{i, k+1}=\Phi_{i}\left(q_{i, k}, x_{i, k}\right)$, where $\Phi_{i}($.$) is a$ smooth function, $x_{i, k} \in \mathcal{X}_{i}$ is the motion control input of the $i^{\text {th }}$ node at time instant $k \geq 1$ and $\mathcal{X}_{i}$ is the set of the admissible control inputs of the $i^{\text {th }}$ node. Then, finding the optimum trajectories of the nodes translates to finding the optimum set of motion control inputs.

\section{B. Probabilistic Characterization of Communication Chan- nels}

Consider a discretized workspace. Each robot constantly updates its decision over this grid and transmits its updated binary decision map to the base station. Consider the case where $(0,1)$ is modulated to $(-1,1)$ at the transmitter. Because of fading, the base station receives a corrupted version of the transmitted decision, given by

$$
z_{i, k}(q)=\sqrt{P_{i, k}} u_{i, k}(q)+w_{i, k},
$$


where $u_{i, k}(q) \in\{-1,1\}$ is the modulated binary decision of the $i^{\text {th }}$ node at time instant $k \geq 1$, regarding the presence of a target at position $q$, and $P_{i, k}>0$ is the instantaneous channel power in the transmission from the $i^{\text {th }}$ node to the base station at time instant $k \geq 1$, which is space-varying. Moreover, $w_{i, k}$ is a zero-mean Gaussian noise with the variance $\sigma_{\mathrm{th}}^{2}$, which represents the receiver thermal noise.

As shown in the communication literature [16], $P_{i, k}$ can be modeled as a multi-scale system with three major dynamics: multipath fading, shadow fading and path loss. Let $P(q)$ denote the channel power in the transmission from a node at $q \in \mathcal{W}$ to the base station, such that $P_{i, k}=P\left(q_{i, k}\right)$. We then have the following characterization for $P(q)$ (in $\mathrm{dB}$ ), using a 2D non-stationary random field model [5], [16]: $P_{\mathrm{dB}}(q)=$ $K_{\mathrm{dB}}-10 n_{\mathrm{PL}} \log _{10}\left(\left\|q-q_{b}\right\|\right)+P_{\mathrm{SH}}(q)+P_{\mathrm{MP}}(q)$, where $P_{\mathrm{dB}}(q)=10 \log _{10}(P(q)), q_{b}$ is the position of the base station, $K_{\mathrm{dB}}$ and $n_{\mathrm{PL}}$ are path loss parameters and $P_{\mathrm{SH}}(q)$ and $P_{\mathrm{MP}}(q)$ are random variables representing the effects of shadow fading and multipath fading in $\mathrm{dB}$ respectively. As an example, Fig. 2 shows the received signal power (which is proportional to channel power) across a route in the basement of ECE building at UNM [17], where the three main dynamics are marked. It can be seen that in

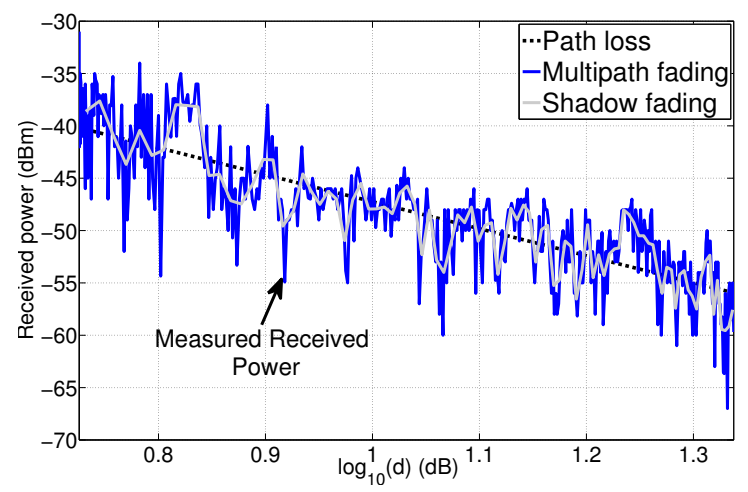

Fig. 2. Underlying dynamics of the wireless channel across a route in the basement of the ECE building [17]. $d$ is the distance to the transmitter.

a realistic communication setting, the spatial variations of the channel can make connectivity maintenance and robust operation challenging.

\section{Multi-Robot Surveillance Using Mobile SENSOR NETWORKS}

In order to find proper communication-aware motion decisions, in this section we derive expressions for the detection performance of a robot and the base station, as a function of channel and sensing qualities. The results are then used in the next section, when devising a framework for communicationaware surveillance.

\section{A. Sequential Detection at Mobile Nodes}

Let $0 \leq \pi_{0}(q) \leq 1$ denote the prior probability that a target exists at position $q \in \mathcal{W}$, in the absence of any observation. Assuming i.i.d observations, we have the following Likelihood Ratio (LR) at the $i^{\text {th }}$ node, given all its observations up to time instant $k \geq 1$ and for any $q \in \mathcal{W}$ : $\bar{L}_{i, k}(q)=\prod_{\ell=1}^{k} L_{i, \ell}(q)$, where

$$
L_{i, \ell}(q)=\exp \left(\frac{2 A y_{i, \ell}(q)-A^{2}}{2 \sigma_{i, \ell}^{2}(q)}\right) .
$$

The optimum decision regarding the presence (or absence) of a target at position $q \in \mathcal{W}$ can then be made as follows: $\bar{L}_{i, k}(q) \underset{H_{0}}{\stackrel{H_{1}}{\gtrless}} \frac{1-\pi_{0}(q)}{\pi_{0}(q)}$. It can be easily confirmed that this is equivalent to the following sequential detection:

$$
L_{i, k}(q) \underset{H_{0}}{\stackrel{H_{1}}{\gtrless}} \frac{1-\pi_{i, k-1}(q)}{\pi_{i, k-1}(q)} .
$$

In this equation, $\pi_{i, k}(q)$ is the updated posterior of the $i^{\text {th }}$ node using its observations up to (and including) time instant $k \geq 1$ and is given by the following recursion:

$$
\pi_{i, k}(q)=\frac{\pi_{i, k-1}(q) L_{i, k}(q)}{\pi_{i, k-1}(q) L_{i, k}(q)+1-\pi_{i, k-1}(q)},
$$

where we set $\pi_{i, 0}(q)=\pi_{0}(q)$ for all $i$. Note that if position $q$ is out of the sensing range of node $i$ at time instant $\ell$, then $L_{i, \ell}(q)=1$, which does not impact the overall detection. Similarly, if $q$ has not been sensed by node $i$ for all the time instants $\ell \leq k$, then $\bar{L}_{i, k}(q)=1$. In this case, the decision regarding the presence of a target is made solely based on the value of the initial prior, i.e. a target will be reported if $\pi_{0}(q)>0.5$. It should be noted that node $i$ only needs to update its decision at time $k \geq 1$, regarding the presence of a target at position $q \in \mathcal{W}$, if $q \in \mathcal{S}_{i, k}$.

1) Analysis of the Performance at Mobile Nodes: Let $\mathcal{I}_{i, k}(q) \triangleq\left\{1 \leq \ell \leq k \mid q \in \mathcal{S}_{i, \ell}\right\}$ denote the set of time instants, up to and including time $k$, when $q \in \mathcal{W}$ has been visited by the $i^{\text {th }}$ node. The local hypothesis testing of the $i^{\text {th }}$ node is then equivalent to

$$
\sum_{\ell \in \mathcal{I}_{i, k}(q)} \frac{A y_{i, \ell}(q)}{\sigma_{i, \ell}^{2}(q)} \underset{H_{0}}{\stackrel{H_{1}}{\gtrless}} \log \left[\frac{1-\pi_{0}(q)}{\pi_{0}(q)}\right]+\sum_{\ell \in \mathcal{I}_{i, k}(q)} \frac{A^{2}}{2 \sigma_{i, \ell}^{2}(q)} .
$$

Let $\pi_{d, i, k}(q)$ and $\pi_{f, i, k}(q)$ denote the corresponding detection and false-alarm probabilities respectively: $\pi_{d, i, k}(q)=$ $\operatorname{Prob}\left\{\bar{L}_{i, k}(q)>\frac{1-\pi_{0}(q)}{\pi_{0}(q)} \mid H_{1}\right\}$ and $\pi_{f, i, k}(q)=$ $\operatorname{Prob}\left\{\bar{L}_{i, k}(q)>\frac{1-\pi_{0}(q)}{\pi_{0}(q)} \mid H_{0}\right\}$. The following can be easily confirmed:

$$
\begin{aligned}
& \pi_{d, i, k}(q)=Q\left(\frac{\log \left[\frac{1-\pi_{0}(q)}{\pi_{0}(q)}\right]-\frac{1}{2} \sum_{\ell \in \mathcal{I}_{i, k}(q)} A^{2} / \sigma_{i, \ell}^{2}(q)}{\sqrt{\sum_{\ell \in \mathcal{I}_{i, k}(q)} A^{2} / \sigma_{i, \ell}^{2}(q)}}\right), \\
& \pi_{f, i, k}(q)=Q\left(\frac{\log \left[\frac{1-\pi_{0}(q)}{\pi_{0}(q)}\right]+\frac{1}{2} \sum_{\ell \in \mathcal{I}_{i, k}(q)} A^{2} / \sigma_{i, \ell}^{2}(q)}{\sqrt{\sum_{\ell \in \mathcal{I}_{i, k}(q)} A^{2} / \sigma_{i, \ell}^{2}(q)}}\right),
\end{aligned}
$$

where $Q(\alpha)=\frac{1}{\sqrt{2 \pi}} \int_{\alpha}^{\infty} e^{-\tau^{2} / 2} d \tau$. The error probability can then be calculated as

$$
\pi_{e, i, k}(q)=\pi_{0}(q)\left(1-\pi_{d, i, k}(q)\right)+\left(1-\pi_{0}(q)\right) \pi_{f, i, k}(q) .
$$


Note that, for the special case of $\mathcal{I}_{i, k}(q)=\emptyset$, we obtain $\pi_{e, i, k}(q)=\min \left\{\pi_{0}(q), 1-\pi_{0}(q)\right\} .^{2}$

\section{B. Optimal Detection at the Base Station}

Let $\mathrm{SNR}_{i, k}$ represent the instantaneous received SNR in the transmission from the $i^{\text {th }}$ node to the base station at time $k: \mathrm{SNR}_{i, k}=P_{i, k} / \sigma_{\mathrm{th}}^{2}$. The base station will drop any received decision with the received SNR below a predefined threshold, denoted by $\mathrm{SNR}_{\mathrm{TH}}$. Define the binary variable $\lambda_{i, k}$ as follows: $\lambda_{i, k} \triangleq\left\{\begin{array}{cc}1 & \mathrm{SNR}_{i, k}>\mathrm{SNR}_{\mathrm{TH}} \\ 0 & \text { otherwise }\end{array}\right.$. Let $\kappa_{i, k}$ denote the last time instant that node $i$ was connected to the base station, up to (and including) time $k \geq 1$ : $\kappa_{i, k}=\max \left\{\{0\} \cup\left\{1 \leq \ell \leq k \mid \lambda_{i, \ell}=1\right\}\right\}$. Note that since $k \geq 1$, we define $\kappa_{i, k}=0$ to indicate the case that the $i^{\text {th }}$ node has not been yet connected to the base station up to time $k \geq 1$, i.e. $\lambda_{i, \ell}=0$ for $\ell=1, \cdots, k$. At any time instant, the base station fuses the last received decisions of all the robots (if available), regarding the presence of a target at position $q \in \mathcal{W}$. Assuming independent received observations from the nodes, we then have the following LR at the base station and at time $k \geq 1: L_{b, k}(q)=\prod_{i=1}^{n} L_{b, i, \kappa_{i, k}}(q)$ and hypothesis testing: $L_{b, k}(q) \underset{H_{0}}{\stackrel{H_{1}}{\gtrless}} \frac{1-\pi_{0}(q)}{\pi_{0}(q)}$. Here, $L_{b, i, \kappa_{i, k}}(q)$ is the LR corresponding to the last received observation from the $i^{\text {th }}$ robot and can be easily shown to be:

$$
L_{b, i, \ell}(q)=\frac{\pi_{d, i, \ell}(q) \exp \left(\frac{2 h_{i, \ell} z_{i, \ell}(q)}{\sigma_{\mathrm{th}}^{2}}\right)+1-\pi_{d, i, \ell}(q)}{\pi_{f, i, \ell}(q) \exp \left(\frac{2 h_{i, \ell} z_{i, \ell}(q)}{\sigma_{\mathrm{th}}^{2}}\right)+1-\pi_{f, i, \ell}(q)},
$$

for $\ell \geq 1$, where $h_{i, \ell}=\sqrt{P_{i, \ell}}$. Note that in case $q \in \mathcal{W}$ has not been visited by the $i^{\text {th }}$ node up to the last time it was connected to the base station, i.e. $q \in \mathcal{W} \backslash \bigcup_{\ell=1}^{\kappa_{i, k}} \mathcal{S}_{i, \ell}$ for $\kappa_{i, k} \geq 1$, then $L_{b, i, \kappa_{i, k}}(q)=1$. We, furthermore, define $L_{b, i, 0}(q) \triangleq 1$ and the expression for $L_{b, k}(q)$ holds if $\kappa_{i, k}=0$ for any $i$.

\section{Mathematical Characterization of the Performance at the Base Station considering both Sensing and Communication Goals}

The trajectories of the nodes affect both their sensing (target detection) and communication qualities. In order to optimize the motion planning accordingly, we need to mathematically characterize the impact of both on the probability of error at the base station. Finding a closed-form expression for the probability of error at the base station, however, is considerably challenging in general. We thus propose to use the Chernoff bound [15], which is typically a tight upper bound on the probability of error, in order to mathematically characterize the performance at the base station. This derivation will then serve as the foundation for devising communication-aware navigation strategies in the next section.

\footnotetext{
${ }^{2}$ Throughout this paper, we traditionally assume that if $\mathcal{I}=\emptyset$, then 1 ) $\prod_{i \in \mathcal{I}} \alpha_{i}=1$, 2) $\sum_{i \in \mathcal{I}} \alpha_{i}=0$ and 3) $\bigcup_{i \in \mathcal{I}} \mathcal{N}_{i}=\emptyset$, where $\alpha_{i} \in \mathbb{R}$ and $\mathcal{N}_{i}$ denotes an arbitrary set.
}

1) Chernoff Bound On the Probability of Error at the Base Station: Let $\mathcal{I}_{k}(q) \triangleq\left\{1 \leq i \leq n \mid q \in \bigcup_{\ell=1}^{\kappa_{i, k}} \mathcal{S}_{i, \ell} \wedge \kappa_{i, k} \geq 1\right\}$ denote the set of the nodes that have been connected to the base station at least once up to time $k \geq 1$ and visited $q \in \mathcal{W}$ at least once before their last connection to the base station. Based on the previous discussion, for any position $q \in \mathcal{W}$, only the nodes in $\mathcal{I}_{k}(q)$ need to be considered for data fusion: $L_{b, k}(q)=\prod_{i \in \mathcal{I}_{k}(q)} L_{b, i, \kappa_{i, k}}(q)$. Then, after a few lines of derivations, we can show that the Chernoff bound on the probability of detection error at the base station is given by

$$
\begin{aligned}
\pi_{e, b, k}(q) & \leq \inf _{0<s<1} \pi_{0}^{s}(q)\left(1-\pi_{0}(q)\right)^{1-s} \\
& \times \underbrace{\prod_{i \in \mathcal{I}_{k}(q)} \Lambda\left(h_{i, \kappa_{i, k}}, \sigma_{\mathrm{th}}, \pi_{d, i, \kappa_{i, k}}(q), \pi_{f, i, \kappa_{i, k}}(q), s\right)}_{\triangleq \tilde{\pi}_{e, b, k}(q, s)},
\end{aligned}
$$

where $\pi_{e, b, k}(q)$ is the exact probability of detection at the base station, $h_{i, \kappa_{i, k}}=\sqrt{P_{i, \kappa_{i, k}}}$ and $\Lambda\left(h, \sigma, \pi_{d}, \pi_{f}, s\right)=$ $\frac{1}{\sqrt{2 \pi} \sigma} \int_{-\infty}^{\infty} e^{-\frac{(z+h) 2}{2 \sigma^{2}}}\left[\pi_{d} e^{\frac{2 z h}{\sigma^{2}}}+\left(1-\pi_{d}\right)\right]^{s}\left[\pi_{f} e^{\frac{2 z h}{\sigma^{2}}}+(1-\right.$ $\left.\left.\pi_{f}\right)\right]^{1-s} \mathrm{~d} z$, for $0<s<1$.

Consider the case where $\mathrm{SNR}_{\mathrm{TH}} \gg$ $\sup _{i, k, q} \max \left\{1, \log \left(1 / \pi_{f, i, k}(q)\right), \log \left(1 /\left(1-\pi_{d, i, k}(q)\right)\right)\right\}$. This assumption can be thought of as requiring that the communication quality be better than the sensing quality (when a node can get connected). Under this assumption, $\Lambda\left(h, \sigma, \pi_{d}, \pi_{f}, s\right)$ can be approximated as follows: $\Lambda\left(h, \sigma, \pi_{d}, \pi_{f}, s\right) \approx \pi_{d}^{s} \pi_{f}^{1-s}+\left(1-\pi_{d}\right)^{s}\left(1-\pi_{f}\right)^{1-s}$, which results in the following:

$$
\begin{aligned}
\tilde{\pi}_{e, b, k}(q, s) & \approx \prod_{i \in \mathcal{I}_{k}(q)}\left[\pi_{d, i, \kappa_{i, k}}^{s}(q) \pi_{f, i, \kappa_{i, k}}^{1-s}(q)\right. \\
& \left.+\left(1-\pi_{d, i, \kappa_{i, k}}(q)\right)^{s}\left(1-\pi_{f, i, \kappa_{i, k}}(q)\right)^{1-s}\right] .
\end{aligned}
$$

As for the exponent $s$, it is common to use $s=0.5$ instead of solving an optimization problem to find the optimal $s$. Case of $s=0.5$ is referred to as Bhattacharyya bound [15]. Note that in case $\mathcal{I}_{k}(q)=\emptyset$, we have $L_{b, k}(q)=1$. Then, the base station reports presence of a target at $q$ if $\pi_{0}(q)>0.5$ and reports otherwise if $\pi_{0}(q)<0.5$. Furthermore, in this case $\pi_{e, b, k}(q)=\min \left\{\pi_{0}(q), 1-\pi_{0}(q)\right\}$, which is equal to its Chernoff bound.

\section{Planning Strategies For Minimizing the Detection Error Probability at the Base Station}

In this section, we propose a communication-aware navigation strategy that aims to minimize the overall probability of detection error at the base station while maximizing the probability that the robots are connected. Devising such communication-aware navigation strategies requires assessing channel quality at places that are not yet visited by the robots. Wireless channels, however, are not fully predictable due to the rapidly-changing dynamics such as multipath fading, as can be seen from Fig. 2. Therefore, in order to address this, we developed a probabilistic channel assessment framework in [5] and [14]. In this framework, a robot 
characterizes a pdf for channel quality in an unvisited location, based on very few channel measurements in the field (possibly collected along its trajectory during operation). We next summarize this probabilistic approach, which will then be utilized in Section IV-B.

\section{A. Probabilistic Modeling and Assessment of the Spatial Variations of a Wireless Channel [5], [14]}

Let $\mathcal{Q}_{i, k}=\left\{q_{1}, \cdots, q_{N}\right\}$ denote the set of the positions corresponding to the small number of channel measurements available to node $i$ at time instant $k$. The channel measurements can be gathered by the $i^{\text {th }}$ node along its trajectory, partly received from other nodes or available through an offline survey of the channel. Note that $\mathcal{Q}_{i, k}$ and the number of available channel measurements are generally time-varying, but we drop the time dependency for the sake of simplicity and label the positions of the samples available to a node at time instant $k$ by $q_{1}, \cdots, q_{N}$. The stacked vector of the channel power measurements (in $\mathrm{dB}$ ), available to the $i^{\text {th }}$ node at time $k$, is then given by the following linear relation: $Y_{i, k}=\Gamma_{i, k} \theta+\Xi_{i, k}+\Omega_{i, k}$, where $\Gamma_{i, k}=\left[\begin{array}{ll}1_{N} & -D_{i, k}\end{array}\right]$ with $1_{N}$ denoting the $N$-dimensional vector of all ones and $D_{i, k}=\left[10 \log _{10}\left(\left\|q_{1}-q_{b}\right\|\right) \cdots 10 \log _{10}\left(\left\|q_{N}-q_{b}\right\|\right)\right]^{\mathrm{T}}$, $\theta=\left[\begin{array}{ll}K_{\mathrm{dB}} & n_{\mathrm{PL}}\end{array}\right]^{\mathrm{T}}, \Xi_{i, k}=\left[P_{\mathrm{SH}}\left(q_{1}\right) \cdots P_{\mathrm{SH}}\left(q_{N}\right)\right]^{\mathrm{T}}$ and $\Omega_{i, k}=$ $\left[\begin{array}{llll}P_{\mathrm{MP}}\left(q_{1}\right) & \cdots & P_{\mathrm{MP}}\left(q_{N}\right)\end{array}\right]^{\mathrm{T}} . P_{\mathrm{SH}}$ and $P_{\mathrm{MP}}$ are the shadow fading and multipath terms in $\mathrm{dB}$, as described in Section IIB. Based on the commonly-used lognormal distribution for shadow fading and its reported exponential spatial correlation [16], $\Xi_{i, k}$ is a zero-mean Gaussian random vector with the covariance matrix $R_{i, k} \in \mathbb{R}^{N}$, where $\left[R_{i, k}\right]_{\ell_{1}, \ell_{2}}=$ $\xi^{2} \exp \left(-\frac{\left\|q_{\ell_{1}}-q_{\ell_{2}}\right\|}{\beta}\right)$ for $1 \leq \ell_{1}, \ell_{2} \leq N$, with $\xi^{2}$ and $\beta$ denoting the variance of shadow fading component in $\mathrm{dB}$ and its decorrelation distance respectively. As for the pdf of multipath fading, distributions such as Rayleigh, Rician, Nakagami and lognormal are shown to match the distribution of multipath fading (in non-dB domain), depending on the environment [16]. Here, we assume lognormal multipath fading, in which case $\Omega_{i, k}$ has a Gaussian distribution. ${ }^{3}$ We also take the elements of $\Omega_{i, k}$ to be uncorrelated. ${ }^{4}$ As a result, $\Omega_{i, k}$ will be a zero-mean Gaussian random vector with covariance $\omega^{2} I_{N}$, where $\omega^{2}$ is the power of multipath fading component and $I_{N}$ is the $N$-dimensional identity matrix. Let $\hat{P}_{\mathrm{dB}, i, k}(q)=\mathbb{E}\left\{P_{\mathrm{dB}}(q) \mid Y_{i, k}\right\}$ and $\delta_{i, k}^{2}(q)=\mathbb{E}\left\{\left(P_{\mathrm{dB}}(q)-\hat{P}_{\mathrm{dB}, i, k}(q)\right)^{2} \mid Y_{i, k}\right\}$ denote the MMSE assessment of channel power at any position $q \in \mathcal{W}$ and its corresponding error variance, based on the measurements available to the $i^{\text {th }}$ node at time $k$. Assuming negligible estimation error for shadow fading and multipath parameters, we have [5], [14]: $\hat{P}_{\mathrm{dB}, i, k}(q)=\gamma^{\mathrm{T}}(q) \hat{\theta}_{i, k}+\hat{\phi}_{i, k}^{\mathrm{T}}(q) \hat{U}_{i, k}^{-1}\left(Y_{i, k}-\right.$ $\left.\Gamma_{i, k} \hat{\theta}_{i, k}\right)$ and $\delta_{i, k}^{2}(q)=\hat{\xi}_{i, k}^{2}+\hat{\omega}_{i, k}^{2}-\hat{\phi}_{i, k}^{\mathrm{T}}(q) \hat{U}_{i, k}^{-1} \hat{\phi}_{i, k}(q)+$

\footnotetext{
${ }^{3}$ Note that Rayleigh, Rician and Nakagami provide a better fit than lognormal in general. However, mathematical derivations are easier with a lognormal distribution.

${ }^{4}$ This is the case whenever the channel samples are far enough comparing to the wavelength [16], which is usually the case.
}

$\left[\gamma(q)-\Gamma_{i, k}^{\mathrm{T}} \hat{U}_{i, k}^{-1} \hat{\phi}_{i, k}(q)\right]^{\mathrm{T}} \Delta_{\theta, i, k}\left[\gamma(q)-\Gamma_{i, k}^{\mathrm{T}} \hat{U}_{i, k}^{-1} \hat{\phi}_{i, k}(q)\right]$, where $\hat{\xi}_{i, k}, \hat{\beta}_{i, k}, \hat{\omega}_{i, k}$ and $\hat{\theta}_{i, k}$ denote the estimated values of $\xi, \beta, \omega$ and $\theta$, based on the available measurements at the $i^{\text {th }}$ node at time $k, \gamma(q)=\left[1-10 \log \left(\left\|q-q_{b}\right\|\right)\right]^{\mathrm{T}}, \hat{U}_{i, k}=$ $\hat{R}_{i, k}+\hat{\omega}_{i, k}^{2} I_{N}, \hat{R}_{i, k}$ denotes the corresponding estimation of $R_{i, k}$ with $\xi$ and $\beta$ replaced by $\hat{\xi}_{i, k}$ and $\hat{\beta}_{i, k}$ respectively,

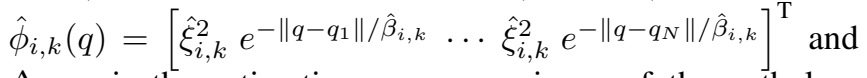
$\Delta_{\theta, i, k}$ is the estimation error covariance of the path loss parameters. ${ }^{5}$ We can then model the channel probabilistically and take the channel power (in $\mathrm{dB}$ ) at any position $q \in \mathcal{W}$ to have a Gaussian distribution with the conditional average and variance of $\hat{P}_{\mathrm{dB}, i, k}(q)$ and $\delta_{i, k}^{2}(q)$ respectively.

\section{B. A Navigation Strategy to Minimize the Detection Error Probability at the Base Station and Maintain the Connectiv- ity of the Mobile Nodes}

Consider the performance analysis of Section III-C. By setting $s=0.5$ (Bhattacharyya bound) and $\pi_{0}(q)=$ $0.5 \forall q \in \mathcal{W}$, we obtain the following approximation for the $\log$ of the Chernoff bound using (11): $\log \left(\widetilde{\pi}_{e, b, k+1}(q, s)\right) \approx$ $\sum_{i \in \mathcal{I}_{k+1}(q)} \log \left(2 \sqrt{\pi_{d, i, \kappa_{i, k+1}}(q)\left(1-\pi_{d, i, \kappa_{i, k+1}}(q)\right)}\right)$. Let $\mathrm{KL}\left(\pi_{d}\right)$ denote the Kullback-Leibler (KL) distance (divergence) between two discrete distributions $\operatorname{Bern}(0.5)$ and $\operatorname{Bern}\left(\pi_{d}\right)$, where $\operatorname{Bern}\left(\pi_{d}\right)$ represents the Bernoulli distribution with the success probability of $\pi_{d}$ [15]. We have $\operatorname{KL}\left(\pi_{d}\right)=-\log \left(2 \sqrt{\pi_{d}\left(1-\pi_{d}\right)}\right)$. Using the definition of $\mathrm{KL}\left(\pi_{d}\right)$ and $\lambda_{i, k}$, we obtain $\log \left(\widetilde{\pi}_{e, b, k+1}(q, s)\right)=-\sum_{i=1}^{n}\left[\lambda_{i, k+1} \operatorname{KL}\left(\pi_{d, i, k+1}(q)\right)+\right.$ $\left.\left(1-\lambda_{i, k+1}\right) \operatorname{KL}\left(\pi_{d, i, \kappa_{i, k}}(q)\right)\right]$, where we define $\pi_{d, i, \kappa_{i, k}}(q)=0.5$ for $\kappa_{i, k}=0$ (the case where the $i^{\text {th }}$ node has not yet been connected to the base station up to and including time $k \geq 1)$. The average of $\log \left(\widetilde{\pi}_{e, b, k+1}(q, s)\right)$, conditioned on the channel values up to (and including) time $k$, is then given by

$$
\begin{aligned}
& \mathbb{E}\left\{\log \left(\widetilde{\pi}_{e, b, k+1}(q, s)\right) \mid \bar{\lambda}_{k}\right\}=-\sum_{i=1}^{n}\left[\mathbb{E}\left\{\lambda_{i, k+1}\right\} \times\right. \\
& \left.\left(\operatorname{KL}\left(\pi_{d, i, k+1}(q)\right)-\operatorname{KL}\left(\pi_{d, i, \kappa_{i, k}}(q)\right)\right)+\operatorname{KL}\left(\pi_{d, i, \kappa_{i, k}}(q)\right)\right],
\end{aligned}
$$

where $\bar{\lambda}_{k}=\left[\lambda_{1,1} \cdots \lambda_{n, k}\right]^{\mathrm{T}}$. Using the probabilistic channel modeling and learning framework of the previous section, we have

$$
\mathbb{E}\left\{\lambda_{i, k+1}\right\}=Q\left(\frac{P_{\mathrm{TH}, \mathrm{dB}}-\hat{P}_{\mathrm{dB}, i, k}\left(q_{i, k+1}\right)}{\delta_{i, k}\left(q_{i, k+1}\right)}\right),
$$

with $P_{\mathrm{TH}, \mathrm{dB}}=10 \log _{10}\left(\sigma_{\mathrm{th}}^{2} \mathrm{SNR}_{\mathrm{TH}}\right)$ denoting the channel power threshold (in $\mathrm{dB}$ ). Note that by applying Chernoff bound approximation, (12) becomes separated into $n$ separate contributions from individual nodes. We then propose the following localized motion objective for the $i^{\text {th }}$ node at time $k$, in order to minimize its contribution to the average

\footnotetext{
${ }^{5} \mathrm{We}$ omit the details of the estimation of the underlying parameters and refer the readers to [5], [14], [18] for more details.
} 
detection error probability of the next time step at the base station:

$$
J_{i, k}\left(q_{i, k+1}\right)=\underbrace{\mathbb{E}\left\{\lambda_{i, k+1}\right\}}_{\text {Comm. obj. }} \underbrace{\left[\int_{\mathcal{W}} \mathrm{KL}\left(\pi_{d, i, k+1}(q)\right) \mathrm{d} q-C_{i, k}\right]}_{\text {Sensing obj. }},
$$

where $C_{i, k} \triangleq \int_{\mathcal{W}} \operatorname{KL}\left(\pi_{d, i, \kappa_{i, k}}(q)\right) \mathrm{d} q$ is a constant (is not a function of $q_{i, k+1}$ ). The optimal control input for node $i$ at time $k$ is then calculated using the following online optimization problem:

$$
\begin{aligned}
& x_{i, k}^{*}=\underset{x_{i, k}}{\operatorname{argmax}} J_{i, k}\left(q_{i, k+1}\right) \\
& \text { s.t. 1) } q_{i, k+1}=\Phi_{i}\left(q_{i, k}, x_{i, k}\right) \text { and 2) } x_{i, k} \in \mathcal{X}_{i} .
\end{aligned}
$$

Note that the sensing part of (14) is always non-negative as $\mathrm{KL}\left(\pi_{d, i, k+1}(q)\right) \geq \operatorname{KL}\left(\pi_{d, i, \kappa_{i, k}}(q)\right)$ for every $q \in \mathcal{W}$.

The motion objective of (15) takes an interesting form that shows 1) the separation of communication and sensing objectives for the purpose of navigation, 2) that solely from a sensing perspective, each node should minimize its surveillance uncertainty by maximizing its $\mathrm{KL}$ distance to $\operatorname{Bern}(0.5), 3)$ that solely from a communication perspective, each node should maximize the percentage of times that it is connected to the base station and 4) that the optimal trajectory is the one that provides the right balance between these objectives. In other words, the localized navigation strategy is the one that minimizes the sensing uncertainty while maximizing the probability of being connected to the base station.

\section{Simulation Results}

In this section we present simulation results to show the performance of the proposed communication-aware surveillance approach. Consider a surveillance scenario where three mobile sensors are tasked with exploring a given environment to detect the possible presence of targets.

We assume the following first-order dynamics for the mobile nodes: $q_{i, k+1}=q_{i, k}+x_{i, k}$, where $\left\|x_{i, k}\right\| \leq d_{\max }$ is the control input. We also consider a distance-dependent sensing model for the mobile nodes, where $\sigma_{i, k}^{2}(q)$ is given by the following:

$$
\sigma_{i, k}^{2}(q)=\left\{\begin{array}{cc}
\nu\left\|q_{i, k}-q\right\|^{2}+\varepsilon & \left\|q_{i, k}-q\right\|<d_{\text {sen }} \\
\infty & \text { otherwise }
\end{array}\right.
$$

where $\nu>0$ and $\varepsilon>0$ are positive constants and $d_{\text {sen }}$ is the sensing radius (sensing range) of the mobile sensors. This model implies that no information will be gathered from areas that are beyond the sensing range of the sensors. At any time instant, the mobile nodes process their recently-gathered data and send their updated map of targets to the base station. The communication channel between each node and the base station is simulated using our probabilistic channel simulator [14]. The nodes assess the channel probabilistically along their trajectories, using the framework of Section IV-A. As more channel measurements become available, the value of $\delta_{i, k}\left(q_{i, k}\right)$ in (13) becomes smaller, resulting in a more dominant communication part. In this example, we assume that the robots start with $1 \%$ a priori channel measurements that are randomly distributed over the environment. We use the following parameters: $d_{\max }=2.0 \mathrm{~m}, A=5, \nu=0.5$, $\varepsilon=1.0, d_{\mathrm{sen}}=3.0 \mathrm{~m}, K_{\mathrm{dB}}=-40 \mathrm{~dB}, n_{\mathrm{PL}}=2, \xi=4 \mathrm{~dB}$, $\beta=4 \mathrm{~m}, \omega=1.9975 \mathrm{~dB}, \sigma_{\text {th }}^{2}=-80 \mathrm{~dB}, \mathrm{SNR}_{\mathrm{TH}}=22 \mathrm{~dB}$ and $\pi_{0}(q)=0.5$.

Fig. 3 shows the communication and sensing parts of the objective function of (14), in a window around one of the robots at a specific time instant. The optimum position for optimizing each individual part is shown by a white cross on the corresponding figure. It can be seen that the optimum position that provides the best sensing objective is considerably different from the one that provides the best communication objective in this example. The overall optimum point is then the one that provides the right balance between these two objectives. Fig. 4 shows the trajectories of the mobile nodes
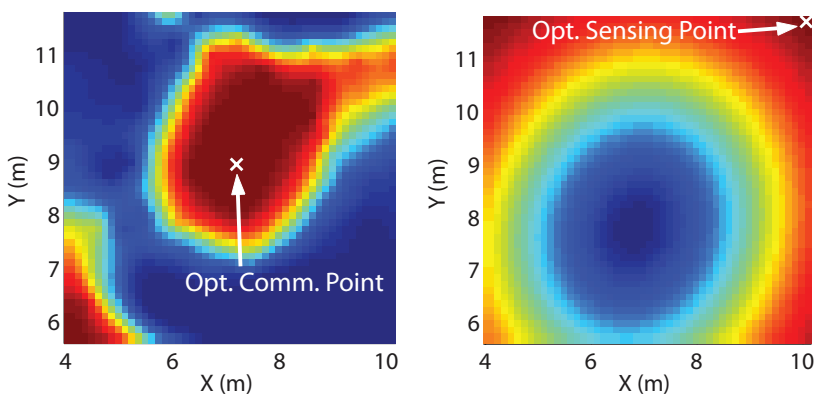

Fig. 3. The communication (left) and sensing (right) parts of the objective function of (14), in a window around one of the robots at a specific time instant. The optimum position for optimizing each individual part is shown by a white cross on the corresponding figure (see the pdf for better visual clarity).

when the localized navigation strategy of (15) is deployed. The dark regions of Fig. 4 represent the positions where the nodes are not connected to the base station. It can be seen that by using the proposed communication-aware navigation strategy, the nodes will gradually converge to the connected regions (if they are not connected to begin with) and maintain their connectivity. In other words, in this case the communication-aware strategy forces the nodes to explore the regions with better link qualities, in order to minimize the next-step detection error probability at the base station.

Finally, Fig. 5 shows the performance of the surveillance at the base station. The plot shows the spatial average of the probability of error at the base station, i.e. $\pi_{k \text {,ave }}=$ $\int_{\mathcal{W}} \pi_{e, b, k}(q) d q /|\mathcal{W}|$, as a function of time. It can be seen that the overall uncertainty decreases as the nodes move. It should be noted that since each robot is required to maintain its connectivity all the time, it is limited in its exploration. This results in an error floor as can be seen from Fig. 5.

\section{CONCLUSiOnS}

In this paper, we considered the problem of communication-aware surveillance, where a number of 


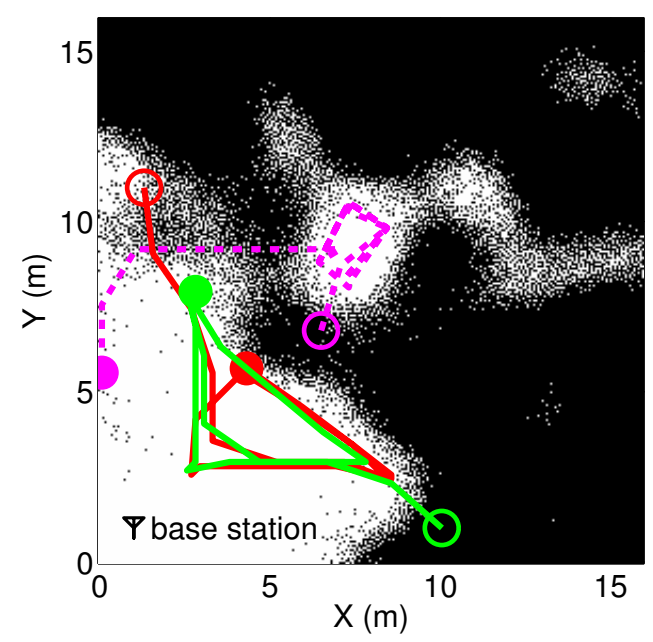

Fig. 4. The trajectories of the three mobile nodes when the localized navigation strategy of (15) is used. The empty circles and the filled ones denote the initial and final positions respectively. The dark regions are areas where the nodes are not connected to the base station. The nodes move along the trajectories that minimize their sensing uncertainty while maintaining their connectivity to the base station (see the pdf for better visual clarity).

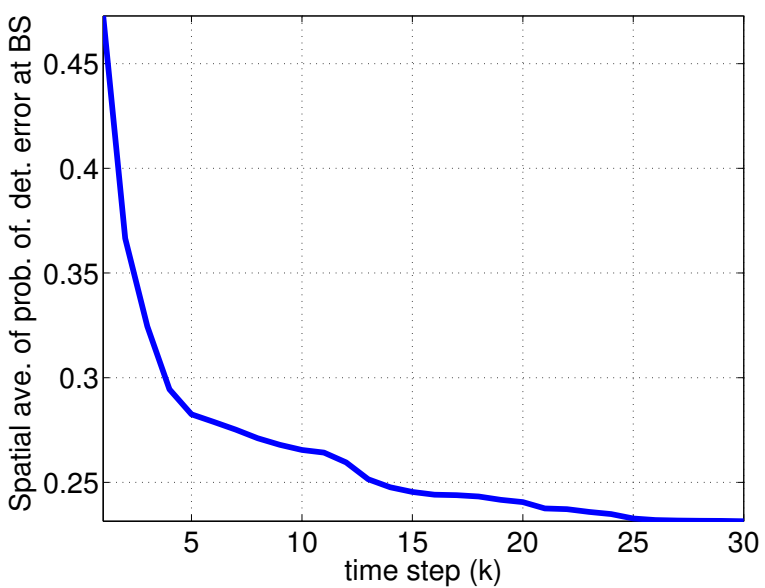

Fig. 5. Spatial average of the probability of detection error at the base station as a function of time.

mobile nodes are tasked with surveying an area to detect the possible presence of targets and reporting their findings back to a fixed base station, in the presence of realistic communication channels. We considered the scenario where the base station needs to be constantly informed of the surveillance result. We showed how to build local motion planning objective functions that integrate sensing goals with communication objectives that are reflective of link qualities in realistic communication environments. More specifically, we showed that the motion planning objective can be separated into a sensing function that maximizes the Kullback-Leibler (KL) divergence between the maximum uncertainty state and the current one, and a communication function, that maximizes the probability of being connected. We then devised motion trajectories that provide the right balance between sensing and communication. We furthermore showed how the probability of connectivity can be assessed online by using our previously-proposed channel assessment framework.

\section{REFERENCES}

[1] I. F. Akyildiz, S. Weilian, Y. Sankarasubramaniam, and E. Cayirci, "A Survey on Sensor Networks," IEEE Communications Magazine, vol. 40, no. 8, pp. 102 - 114, Aug. 2002.

[2] V. Kumar, D. Rus, and S. Singh, " Robot and Sensor Networks for First Responders ," IEEE Pervasive Computing, pp. 24-33, OctoberDecember 2004.

[3] J. Cortés, S. Martínez, T. Karatas, and F. Bullo, "Coverage control for mobile sensing networks," IEEE Transactions on Robotics and Automation, vol. 20, no. 2, pp. 243-255, 2004.

[4] P. E. Rybski, N. P. Papanikolopoulos, S. A. Stoeter, D. G. Krantz, K. B. Yesin, M. Gini, R. Voyles, D. F. Hougen, B. Nelson, and M. D. Erickson, "Enlisting rangers and scouts for reconnaissance and surveillance," IEEE Robotics Automation Magazine, vol. 7, no. 4, pp. $14-24$, Dec. 2000.

[5] A. Ghaffarkhah and Y. Mostofi, "Channel Learning and Communication-Aware Motion Planning in Mobile Networks," in Proceedings of the American Control Conference (ACC), Baltimore, MD, June 2010, pp. 5413-5420.

[6] - "Communication-Aware Motion Planning in Mobile Networks," April 2010, submitted, IEEE Transactions on Automatic Control, special issue on Wireless Sensor and Actuator Networks.

[7] M. Lindhe and K. H. Johansson, "Adaptive Exploitation of Multipath Fading for Mobile Sensors," in Proceedings of IEEE International Conference on Robotics and Automation (ICRA), Anchorage, AK, 2010

[8] S. Kumar, Z. Feng, and D. Shepherd, "Collaborative Signal and Information Processing in Microsensor Networks," IEEE Signal Processing Magazine, vol. 19, no. 2, pp. 13 -14, Mar. 2002.

[9] B. Chen, R. Jiang, T. Kasetkasem, and P. K. Varshney, "Channel Aware Decision Fusion in Wireless Sensor Networks," IEEE Transactions on Signal Processing, vol. 52, no. 12, pp. 3454 - 3458, dec. 2004.

[10] B. Liu and B. Chen, "Decentralized Detection in Wireless Sensor Networks with Channel Fading Statistics," EURASIP J. Wirel. Commun Netw., vol. 2007, no. 1, pp. 11-11, 2007.

[11] J. Cortés, S. Martínez, and F. Bullo, "Spatially-distributed coverage optimization and control with limited-range interactions," ESAIM: Control, Optimisation and Calculus of Variations, vol. 11, pp. 691719, 2005.

[12] Y. Wang and I. Hussein, "Awareness Coverage Control Over LargeScale Domains With Intermittent Communications," IEEE Transactions on Automatic Control, vol. 55, no. 8, pp. 1850 -1859, 2010.

[13] B. Grocholsky, J. Keller, V. Kumar, and G. Pappas, " Cooperative Air-Ground Surveillance ," IEEE Robotics and Automation Magazine, vol. 13, no. 3, pp. 16-25, 2006.

[14] Y. Mostofi, M. Malmirchegini, and A. Ghaffarkhah, "Estimation of Communication Signal Strength in Robotic Networks," in Proceedings of IEEE International Conference on Robotics and Automation (ICRA), Anchorage, AK, May 2010, pp. 1946 -1951.

[15] H. V. Poor, An Introduction to Signal Detection and Estimation (2nd ed.). New York, NY, USA: Springer-Verlag New York, Inc., 1994.

[16] A. Goldsmith, Wireless Communications. Cambridge University Press, 2005

[17] Y. Mostofi, A. Gonzales-Ruiz, A. Ghaffarkhah, and D. Li, "Characterization and Modeling of Wireless Channels for Networked Robotic and Control Systems - A Comprehensive Overview," in Proceedings of 2009 IEEE/RSJ International Conference on Intelligent Robots and Systems (IROS), St. Louis, MO, October 2009.

[18] M. Malmirchegini and Y. Mostofi, "On the Spatial Predictability of Communication Channels," October 2010, submitted, IEEE Transactions on Wireless Communications. 